This item was submitted to Loughborough's Research Repository by the author.

Items in Figshare are protected by copyright, with all rights reserved, unless otherwise indicated.

\title{
Shared-control for a UAV operating in the 3D space
}

PLEASE CITE THE PUBLISHED VERSION

https://doi.org/10.1109/ecc.2015.7330771

PUBLISHER

IEEE @ EUCA

VERSION

AM (Accepted Manuscript)

LICENCE

CC BY-NC-ND 4.0

REPOSITORY RECORD

Jiang, Jingjing, and Alessandro Astolfi. 2019. "Shared-control for a UAV Operating in the 3D Space". figshare. https://hdl.handle.net/2134/36762. 


\title{
Shared-Control for a UAV Operating in the 3D Space
}

\author{
Jingjing Jiang $^{1}$ and Alessandro Astolfi ${ }^{2}$
}

\begin{abstract}
This paper presents a shared-control scheme for a UAV moving in a 3D space while its feasible Cartesian position set is defined by a group of linear inequalities. A hysteresis switch is used to combine the human input and the feedback control input based on the definitions of a safe set, a hysteresis set and a "dangerous" set. Case studies given in the paper show the effectiveness of the shared-control algorithm.
\end{abstract}

\section{INTRODUCTION}

Unmanned Aerial Vehicles (UAVs), commonly known as drones, are aircrafts without a human pilot aboard. They are used in military attacks [1] and surveillance [2], [3] and have recently received significant attentions. Business report states that the global UAV market size can be expected to grow to over 8 billion dollars by 2018. Nowadays UAVs not only play a role in military applications [4], [5], but are also widely used in civil applications, such as policing [6], weather determination [7], mapping [8] and agriculture [9].

Various traditional methods to control the positions of a UAV, such as PI controls [10], linearized controls [11] and fuzzy controls [12], [13] have been used. Even though these controllers are easy to design and implement, they heavily depend on designer's experience. In addition, the assumption of the availability of the dynamics of the UAV is not always practical. Therefore [14] has introduced a nonlinear controller using neural network to online learn the dynamics of the UAV and has proved that the tracking errors and the estimation errors are semi-globally uniformly ultimately bounded, while [15] has utilized an adaptive controller based on backstepping to compensate the uncertainty of the UAV and has proved that the tracking errors in the $x, y, z$ variables and in the yaw rotation asymptotically converge to zero.

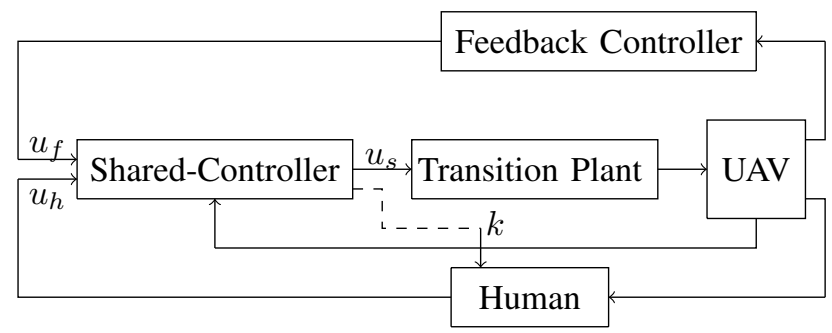

Fig. 1: The Proposed Control Framework

\footnotetext{
${ }^{1}$ J. Jiang is with the Department of Electrical and Electronic Engineering, Imperial College London, UK, E-mail: jingjing.jianglo@imperial.ac.uk

${ }^{2}$ A. Astolfi is with the Dept. of Electrical and Electronic Engineering, Imperial College London, London, SW7 2AZ, UK and the DICII, University of Roma "Tor Vergata", Via del Politecnico 1, 00133 Rome, Italy, E-mail: a.astolfieimperial.ac.uk
}

This paper does not focus on the angular dynamics of a UAV (i.e. the dynamics of the pitch angle, the yaw angle and the roll angle). Instead, we assume the dynamics of the overall open loop system (including the 'Transition Plant' which will be described later in this paragraph and the 'UAV' block in Figure 1) can be described by the equations

$$
\begin{aligned}
& \dot{x}=v_{s} \cos \phi \cos \theta, \\
& \dot{y}=v_{s} \cos \phi \sin \theta, \\
& \dot{z}=v_{s} \sin \phi, \\
& \dot{\theta}=\omega_{1 s}, \\
& \dot{\phi}=\omega_{2 s},
\end{aligned}
$$

where $(x, y, z)$ denotes the Cartesian coordinates of the UAV center of mass, $\theta$ is the heading angle (from East) of the UAV, $\phi$ represents the UAV path angle (from the horizontal), $v_{s}$ is the forward speed, $\omega_{1 s}$ and $\omega_{2 s}$ denote the angular velocity with respect to the $\theta$ and $\phi$ axes, respectively. Note that $\left[v_{s}, \omega_{1 s}, \omega_{2 s}\right]^{T}$ is the external input of the system (1). The 'Transition Plant' in Figure 1 is used to transmit the sharedcontrol input $u_{s}$ into the inputs of the UAV, while keeping $u_{s}$ numerically equal to $\left[v_{s}, \omega_{1 s}, \omega_{2 s}\right]^{T}$ and the 'SharedController' is used to combine the human action, denoted by 'Human', and the 'Feedback Controller'. Note that the name "shared-control' used in the paper has the same meaning as that in the anti-lock braking system. Shared-control has significant superiorities over human control since the safety of the system is guaranteed: we use Lyapunov-like analysis to prove the stability of the closed-loop system which satisfies all constraints at all times. On the other hand, compared with automatic control, the human operator could develop his/her skills using the shared-controller and be self-confident about his/her activities, thereby enjoying the fun of driving. The function $k$ in Figure 1 provides a feedback signal to the human indicating how dangerous the situation is.

[16] has presented a shared-control algorithm for the kinematic model of a mobile robot. This paper extends the results in [16] to a 3D space and provides formal proofs of the properties of the closed-loop system with the shared-control law. Furthermore, new case studies on 3D trajectory tracking is given in the paper.

This paper is organized as follows. Section II formulates the problem and gives definitions and assumptions. One solution to the shared-control problem is presented in Section III, in which formal properties of the shared-controller are provided. One numerical example is given in Section IV to show the effectiveness of the proposed shared-control strategy. Finally, Section V gives some conclusions and ideas for future work. 


\section{PROBLEM STATEMENT AND ASSUMPTIONS}

In this section we formulate the shared-control problem for the UAV model described by equations (1).

Let

$$
\begin{array}{r}
{\left[\begin{array}{c}
v_{s} \\
\omega_{1 s} \\
\omega_{2 s}
\end{array}\right]=\left[1-k\left(x, y, z, \theta, \phi, v_{h}\right)\right]\left[\begin{array}{c}
v_{f} \\
\omega_{1 f} \\
\omega_{2 f}
\end{array}\right]} \\
+k\left(x, y, z, \theta, \phi, v_{h}\right)\left[\begin{array}{c}
v_{h} \\
\omega_{1 h} \\
\omega_{2 h}
\end{array}\right],
\end{array}
$$

where $u_{f}=\left[v_{f}, \omega_{1 f}, \omega_{2 f}\right]^{T}$, denoted as $f$-control, represents the feedback-control action and $u_{h}=\left[v_{h}, \omega_{1 h}, \omega_{2 h}\right]^{T}$, denoted as $h$-control, describes the human action. We use the name s-closed-loop to denote the system described by equation (1) with the input given by the equation (2), and the name $h$-closed-loop to denote the system described by the equations

$$
\begin{aligned}
& \dot{x}=v_{h} \cos \phi \cos \theta, \\
& \dot{y}=v_{h} \cos \phi \sin \theta, \\
& \dot{z}=v_{h} \sin \phi, \\
& \dot{\theta}=\omega_{1 h}, \\
& \dot{\phi}=\omega_{2 h} .
\end{aligned}
$$

In addition, $\Omega_{h}$ and $\Omega_{s}$ are used to denote the $\Omega$-limit set (the definition of which is given in [17]) of the h-closed-loop system and of the s-closed-loop system, respectively. Note that the h-closed-loop system and the s-closed-loop system share the same state space $\mathcal{P} \times \mathcal{H} \times \mathcal{A}=\mathbb{R}^{3} \times \mathbb{S} \times \mathbb{A}^{1}$, where $\mathcal{P}$ is the set of Cartesian positions, $\mathcal{H}$ represents the set of heading angles and $\mathcal{A}$ denotes the set of path angles. Let $\mathcal{P}_{a} \in \mathcal{P}$ be a given and compact set describing the admissible Cartesian positions of the system (1) and $u_{h}$ be a given $\mathrm{h}-$ control. Then the shared-control problem can be posed as follows.

Given the system (1), an admissible configuration set $\mathcal{P}_{a}$ and an h-control $u_{h}$, find (if possible)

- an f-control $u_{f}$;

- a sharing function $k$;

- a safe set $\mathcal{R}_{s}\left(v_{h}\right) \triangleq \mathcal{P}_{a} \times \mathcal{H}_{s} \times \mathcal{A}_{s}{ }^{2} \subset \mathcal{P}_{a} \times \mathcal{H} \times \mathcal{A} \triangleq$ $\mathcal{R}\left(v_{h}\right)$;

such that the s-closed-loop system (1)-(2) has the following properties.

\footnotetext{
${ }^{1} \mathbb{A}$ is the set

$$
\mathbb{A}=\left\{\phi \in \mathbb{R}:|\phi| \leq \frac{\pi}{2}\right\}
$$

${ }^{2}$ For any given sufficiently short $\Delta t>0$, any $p(t)=$ $[x(t), y(t), z(t)]^{T} \in \mathcal{P}_{a}$ and any fixed $v_{h}$, the sets $\mathcal{A}_{s}$ and $\mathcal{H}_{s}$ are defined
} as

$\mathcal{A}_{s} \times \mathcal{H}_{s}=\left\{\theta \times \phi \in \mathbb{S} \times \mathbb{A}:\left(p(t)+\Delta t\left[\begin{array}{c}v_{h} \cos \phi \cos \theta \\ v_{h} \cos \phi \sin \theta \\ v_{h} \sin \phi\end{array}\right]\right) \in \mathcal{P}_{a}\right\}$.

for $j \in\{1,2,3\}$, where $r$ is a positive constant, $h_{j}^{i}=$ $-(r+\epsilon)+\sqrt{r^{2}-\left[(\sqrt{2}-1) r-\epsilon-q_{d_{j}}^{i}\right]^{2}}$ and $q_{d}^{i}=$ $\left[q_{d_{1}}^{i}, q_{d_{2}}^{i}, q_{d_{3}}^{i}\right]^{T}=S^{i} p_{d}+T^{i}$. Note that $q_{r_{j}}^{i}<0$ and that
P1) For any initial state $(x, y, z, \theta, \phi) \in \mathcal{R}$, the state $(x(t), y(), z(t), \theta(t), \phi(t))$ for the s-closed-loop system remains in $\mathcal{R}$ for all $t \geq 0$.

P2)

$$
\Omega_{s}= \begin{cases}\Omega_{h} & \text { if } \Omega_{h} \subset \mathcal{R}_{s}\left(v_{h}\right), \\ \Pi_{\mathcal{R}_{s}}\left(\Omega_{h}\right) & \text { if } \Omega_{h} \not \subset \mathcal{R}_{s}\left(v_{h}\right),\end{cases}
$$

where $\Pi_{\mathcal{R}_{s}}\left(\Omega_{h}\right)$ is the projection of $\Omega_{h}$ into the set $\mathcal{R}_{s}\left(v_{h}\right)$, which will be defined in Section III-A.

P3) $u_{s}=u_{h}$ if $(x, y, z, \theta, \phi) \in \mathcal{R}_{s}\left(v_{h}\right)$.

Assumption 1: The non-empty admissible configuration set $\mathcal{P}_{a}$ is defined by a group of linear inequalities, namely

$$
\mathcal{P}_{a}=\left\{p \in \mathbb{R}^{3} \mid S p+T \leq 0\right\},
$$

where $p=[x, y, z]^{T}, S=\left[s_{1}^{T}, s_{2}^{T}, \ldots, s_{m}^{T}\right]^{T} \in \mathbb{R}^{m \times 3}$ and $T=\left[t_{1}, t_{2}, \ldots, t_{m}\right]^{T} \in \mathbb{R}^{m}$. In addition, if $m>3$ then the matrices $S$ and $T$ are such that

$$
\operatorname{rank}\left(\left[\begin{array}{l}
s_{r_{1}} \\
\vdots \\
s_{r_{l}}
\end{array}\right]\right)<\operatorname{rank}\left(\left[\begin{array}{ll}
s_{r_{1}} & t_{r_{1}} \\
\vdots & \vdots \\
s_{r_{l}} & t_{r_{l}}
\end{array}\right]\right),
$$

for all $l \in[4, m]$ and $r_{1}, r_{2}, \ldots, r_{l} \in\{1,2, \ldots, m\}$.

Assumption 2: The reference trajectory in the space $\mathcal{P}$ is continuous.

The above assumptions hold for the rest of the paper.

\section{SHARED-CONTROL DESIGN}

\section{A. Design of the f-control}

As detailed in [18] we design the feedback controller under the assumption that $m=3$. Note that $N_{c}$ f-controls need to be designed and in general, $N_{c} \leq\left(\begin{array}{c}m \\ 2\end{array}\right)$. Consider the $i^{\text {th }}$ group of constraints

$$
S^{i} p+T^{i} \leq 0
$$

where $p=[x, y, z]^{T}, S^{i}=\left[s_{1}^{i^{T}}, s_{2}^{i^{T}}, s_{3}^{i^{T}}\right]^{T} \in \mathbb{R}^{3 \times 3}$ and $T=\left[t_{1}^{i}, t_{2}^{i}, t_{3}^{i}\right]^{T} \in \mathbb{R}^{3}$. Define a new variable $q^{i}$ as $q^{i}=$ $S^{i} p+T^{i}$. To remove the constraint on $q^{i}$, we define a new variable $m^{i}=\left[m_{1}^{i}, m_{2}^{i}, m_{3}^{i}\right]^{T}$, with

$$
m_{j}^{i}=\log \frac{q_{j}^{i}}{q_{r_{j}}^{i}},
$$

for all $j \in\{1,2,3\}$, where $q_{r_{j}}^{i}$ is the reference trajectory defined as

$$
q_{r_{j}}^{i}= \begin{cases}q_{d}^{i}, & \text { if } q_{d_{j}}^{i} \leq\left(1-\frac{\sqrt{2}}{2}\right) r-\epsilon, \\ -\epsilon, & \text { if } q_{d_{j}}^{i} \geq(\sqrt{2}-1) r-\epsilon, \\ h_{j}^{i}, & \text { otherwise },\end{cases}
$$


$q_{r_{j}}^{i}$ is a smooth function. Therefore $\dot{q}_{r}^{i}$ and $\ddot{q}_{r}^{i}$ exist. Let $p_{r}^{i}$, $\alpha_{r}^{i}, v_{r}^{i}, \theta_{r}^{i}, \phi_{r}^{i}$ and $\omega_{r}^{i}$ be given by ${ }^{3}$

$$
\begin{array}{rlrl}
p_{r}^{i} & =S^{i-1}\left(q_{r}^{i}-T^{i}\right), & \alpha_{r}^{i}=S^{i-1} \dot{q}_{r}^{i}, \\
v_{r}^{i}=\sqrt{\alpha_{r_{1}}^{i}+\alpha_{r_{2}}^{i}+\alpha_{r_{3}}^{i}{ }^{2}}, & \theta_{r}^{i}=\operatorname{atan}\left(\alpha_{r_{2}}^{i}, \alpha_{r_{1}}^{i}\right), \\
\phi_{r}^{i}=\operatorname{atan} 2\left(\alpha_{r_{3}}^{i}, \sqrt{{\alpha_{r_{1}}^{i}}^{2}+\alpha_{r_{2}}^{i}}\right), & \omega_{r}^{i}=\left[\dot{\theta}_{r}^{i}, \dot{\phi}_{r}^{i}\right]^{T} .
\end{array}
$$

Suppose $\left(p_{d}, \theta_{d}, \phi_{d}\right) \in \Omega_{h}$. Then the projection of $\left(p_{d}, \theta_{d}, \phi_{d}\right)$ into $\mathcal{R}_{s}\left(v_{h}\right)$ relative to the $i^{\text {th }}$ group of active constraints, i.e. $\Pi_{\mathcal{R}_{s}}^{i}\left(p_{d}, \theta_{d}, \phi_{d}\right)$, is defined as

$$
\Pi_{\mathcal{R}_{s}}^{i}\left(p_{d}, \theta_{d}, \phi_{d}\right)=\left(p_{r}^{i}, \theta_{r}^{i}, \phi_{r}^{i}\right),
$$

where $\left(p_{r}^{i}, \theta_{r}^{i}, \phi_{r}^{i}\right)$ is given by (7). Therefore the projection of $\Omega_{h}$ into $\mathcal{R}_{s}\left(v_{h}\right)$, relative to the $i^{\text {th }}$ group of constraints, is defined by

$$
\Pi_{\mathcal{R}_{s}}^{i}\left(\Omega_{h}\right)=\left\{s \in \mathcal{R}_{s} \mid s=\Pi_{\mathcal{R}_{s}}^{i}\left(p_{d}, \theta_{d}, \phi_{d}\right)\right\},
$$

for all $\left(p_{d}, \theta_{d}, \phi_{d}\right) \in \Omega_{h}$.

With the use of the new variable $m^{i}$ defined in (5), the system (1) can be rewritten as

$$
\begin{aligned}
\dot{m}_{1}^{i} & =\frac{v_{f}^{i} \cos \phi^{i} \cos \theta^{i}}{e^{m_{1}^{i}} q_{r_{1}}^{i}}-\frac{v_{r}^{i} \cos \phi_{r}^{i} \cos \theta_{r}^{i}}{q_{r_{1}}^{i}}, \\
\dot{m}_{2}^{i} & =\frac{v_{f}^{i} \cos \phi^{i} \sin \theta^{i}}{e^{m_{1}^{i}} q_{r_{2}}^{i}}-\frac{v_{r}^{i} \cos \phi_{r}^{i} \sin \theta_{r}^{i}}{q_{r_{2}}^{i}} \\
\dot{m}_{3}^{i} & =\frac{v_{f}^{i} \sin \phi^{i}}{e^{m_{2}^{i}} q_{r_{3}}^{i}}-\frac{v_{r}^{i} \sin \phi_{r}^{i}}{q_{r_{3}}^{i}}, \\
\dot{\theta}^{i} & =\omega_{1 f}^{i} \\
\dot{\phi}^{i} & =\omega_{2 f}^{i} .
\end{aligned}
$$

Let

$$
\theta^{i *}=\operatorname{atan}\left(l_{2}, l_{1}\right), \phi^{i *}=\operatorname{atan}\left(l_{3}, \sqrt{l_{1}^{2}+l_{2}^{2}}\right)
$$

where $\gamma_{1}>0, \gamma_{2}>0, \gamma_{3}>0$ and

$$
\begin{array}{r}
l_{1}=e^{m_{1}^{i}}\left(v_{r}^{i} \cos \phi_{r}^{i} \cos \theta_{r}^{i}+\gamma_{1} l_{1}\right), \\
l_{2}=e^{m_{2}^{i}}\left(v_{r}^{i} \cos \phi_{r}^{i} \sin \theta_{r}^{i}+\gamma_{2} l_{2}\right), \\
l_{3}=e^{m_{3}^{i}}\left(v_{r}^{i} \sin \phi_{r}^{i}+\gamma_{3} l_{3}\right) .
\end{array}
$$

Note that if $\left|\phi^{i *}\right|=\frac{\pi}{2}$, then $\theta^{i *}=\operatorname{atan}(0,0)$, which is not uniformly defined. In this case, we define $\theta^{i *}=\theta_{r}$. In addition, $\phi^{i *}$ and $\theta^{i *}$ are reference angles for $\phi^{i}$ and $\theta^{i}$, respectively.

Consider the Lyapunov function associated with the $i^{\text {th }}$ group of active constraints given by

$L^{i}\left(m_{1}^{i}, m_{2}^{i}, m_{3}^{i}, \theta^{i}, \phi^{i}\right)=\frac{1}{2}\left[\begin{array}{c}\left(\theta^{i}-\theta^{i *}\right)^{2}+\left(\phi^{i}-\phi^{i *}\right)^{2} \\ +m_{1}^{i^{2}}+m_{2}^{i^{2}}+m_{3}^{i^{2}}\end{array}\right]$,

\footnotetext{
${ }^{3}$ We use the 4 quadrant arctan function.
}

and choose $u_{f}^{i}=\left[v_{f}^{i}, \omega_{1 f}^{i}, \omega_{2 f}^{i}\right]^{T}$ such that $L^{i}<0$ for all $\left(m_{1}^{i}, m_{2}^{i}, m_{3}^{i}, \theta^{i}, \phi^{i}\right) \neq\left(0,0,0, \theta^{i *}, \phi^{i *}\right)$. One such a choice is given as

$$
\begin{aligned}
& v_{f}^{i}=\sqrt{l_{1}^{2}+l_{2}^{2}+l_{3}^{2}}, \\
& \omega_{1 f}^{i}=\dot{\theta}^{i *}+\frac{m_{1}^{i} v_{f}^{i} \cos \phi^{i}}{e^{m_{1}^{i}} q_{r_{1}}^{i}} \sin \frac{\theta^{i}+\theta^{i *}}{2} \operatorname{sinc} \frac{\theta^{i}-\theta^{i *}}{2} \\
& -\frac{m_{2}^{i} v_{f}^{i} \cos \phi^{i}}{e^{m_{2}^{i}} q_{r_{2}}^{i}} \cos \frac{\theta^{i}+\theta^{i *}}{2} \operatorname{sinc} \frac{\theta^{i}-\theta^{i *}}{2} \\
& -\gamma_{4}\left(\theta^{i}-\theta^{i *}\right) \text {, } \\
& \omega_{2 f}^{i}=\dot{\phi}^{i *}+\frac{m_{1}^{i} v_{f}^{i} \cos \theta^{i *}}{e^{m_{1}^{i}} q_{r_{1}}^{i}} \sin \frac{\phi^{i}+\phi^{i *}}{2} \operatorname{sinc} \frac{\phi^{i}-\phi^{i *}}{2} \\
& -\frac{m_{2}^{i} v_{f}^{i} \sin \theta^{i *}}{e^{m_{2}^{i}} q_{r_{2}}^{i}} \sin \frac{\phi^{i}+\phi^{i *}}{2} \operatorname{sinc} \frac{\phi^{i}-\phi^{i *}}{2} \\
& -\frac{m_{3}^{i} v_{f}^{i}}{e^{m_{3}^{i}} q_{r_{3}}^{i}} \cos \frac{\phi^{i}+\phi^{i *}}{2} \operatorname{sinc} \frac{\phi^{i}-\phi^{i *}}{2}-\gamma_{5}\left(\phi^{i}-\phi^{i *}\right),
\end{aligned}
$$

which yields

$$
\begin{aligned}
\dot{L}^{i}= & \gamma_{1} \frac{m_{1}^{i^{2}}}{q_{r_{1}}^{i}}+\gamma_{2} \frac{m_{2}^{i^{2}}}{q_{r_{2}}^{i}}+\gamma_{3} \frac{m_{1}^{i^{2}}}{q_{r_{1}}^{i}}-\gamma_{4}\left(\theta^{i}-\theta^{i *}\right)^{2} \\
& -\gamma_{5}\left(\phi^{i}-\phi^{i *}\right)^{2} \leq 0 .
\end{aligned}
$$

This can be pushed back into the $(p, \theta, \phi)$ coordinates yielding

$$
\begin{aligned}
& v_{f}^{i}= \begin{array}{c}
\left(\frac{q_{1}^{i}}{q_{r_{1}}^{i}}\right)^{2}\left(v_{r}^{i} \cos \phi_{r}^{i} \cos \theta_{r}^{i}+\gamma_{1} \log \frac{q_{1}^{i}}{q_{r_{1}}^{i}}\right)^{2} \\
+\left(\frac{q_{2}^{i}}{q_{r_{2}}^{i}}\right)^{2}\left(v_{r}^{i} \cos \phi_{r}^{i} \sin \theta_{r}^{i}+\gamma_{2} \log \frac{q_{2}^{i}}{q_{r_{2}}^{i}}\right)^{2} \\
+\left(\frac{q_{3}^{i}}{q_{r_{3}}^{i}}\right)^{2}\left(v_{r}^{i} \sin \phi_{r}^{i}+\gamma_{3} \log \frac{q_{3}^{i}}{q_{r_{3}}^{i}}\right)^{2}
\end{array} \\
& \omega_{1 f}^{i}= \dot{\theta}^{i *}+\frac{q_{1}^{i}}{\log \frac{q_{r_{1}}^{i}}{q_{f}^{i}} \cos \phi^{i}} \sin \frac{\theta^{i}+\theta^{i *}}{2} \operatorname{sinc} \frac{\theta^{i}-\theta^{i *}}{2} \\
&-\frac{q_{1}^{i}}{\log \frac{q_{2}^{i}}{q_{r_{2}}^{i}} v_{f}^{i} \cos \phi^{i}} \\
&-\gamma_{4}\left(\theta^{i}-\theta^{i *}\right), \\
&
\end{aligned}
$$

$$
\begin{aligned}
\omega_{2 f}^{i}= & \dot{\phi}^{i *}+\frac{\log \frac{q_{1}^{i}}{q_{r_{1}}^{i}} v_{f}^{i} \cos \theta^{i *}}{q_{1}^{i}} \sin \frac{\phi^{i}+\phi^{i *}}{2} \operatorname{sinc} \frac{\phi^{i}-\phi^{i *}}{2} \\
& -\frac{\log \frac{q_{2}^{i}}{q_{r_{2}}^{i}} v_{f}^{i} \sin \theta^{i *}}{q_{2}^{i}} \sin \frac{\phi^{i}+\phi^{i *}}{2} \operatorname{sinc} \frac{\phi^{i}-\phi^{i *}}{2} \\
& -\frac{\log \frac{q_{3}^{i}}{q_{r_{3}}^{i}} v_{f}^{i}}{q_{3}^{i}} \cos \frac{\phi^{i}+\phi^{i *}}{2} \operatorname{sinc} \frac{\phi^{i}-\phi^{i *}}{2} \\
- & \gamma_{5}\left(\phi^{i}-\phi^{i *}\right),
\end{aligned}
$$


where

$$
\begin{aligned}
& \theta^{i *}=\operatorname{atan}\left(\begin{array}{c}
\frac{q_{2}^{i}}{q_{r_{2}}^{i}}\left(v_{r}^{i} \cos \phi_{r}^{i} \sin \theta_{r}^{i}+\gamma_{2} \log \frac{q_{2}^{i}}{q_{r_{2}}^{i}}\right), \\
\frac{q_{1}^{i}}{q_{r_{1}}^{i}}\left(v_{r}^{i} \cos \phi_{r}^{i} \cos \theta_{r}^{i}+\gamma_{1} \log \frac{q_{1}^{i}}{q_{r_{1}}^{i}}\right)
\end{array}\right), \\
& \phi^{i *}=\operatorname{atan}\left(\begin{array}{c}
\frac{q_{3}^{i}}{q_{r_{3}}^{i}}\left(v_{r}^{i} \sin \phi_{r}^{i}+\gamma_{3} \log \frac{q_{3}^{i}}{q_{r_{3}}^{i}}\right), \\
\frac{q_{1}^{i}}{q_{r_{1}}^{i}}\left(v_{r}^{i} \cos \phi_{r}^{i} \cos \theta_{r}^{i}+\gamma_{1} \log \frac{q_{1}^{i}}{q_{r_{1}}^{i}}\right) \\
+\frac{q_{2}^{i}}{q_{r_{2}}^{i}}\left(v_{r}^{i} \cos \phi_{r}^{i} \sin \theta_{r}^{i}+\gamma_{2} \log \frac{q_{2}^{i}}{q_{r_{2}}^{i}}\right)
\end{array}\right)
\end{aligned}
$$

and $q_{j}^{i}=s_{j}^{i} p+t_{j}^{i}, q_{r_{j}}^{i}=s_{j}^{i} p_{r}+t_{j}^{i}$ for all $j \in\{1,2,3\}$. Note that $v_{f}^{i} \geq 0$. This is consistent with its physical meaning: the speed of a UAV is always nonnegative.

Lemma 1: Consider the system (1) controlled by (11) where $q_{r}^{i}$ is defined by (6) and $v_{r}^{i}, \theta_{r}^{i}$ and $\phi_{r}^{i}$ are given by (7). Suppose $\mathcal{P}_{a}$ is described by (3), $(x(0), y(0), z(0)) \in \mathcal{P}_{a}$ and the time that a feedback controller is active is larger than $\Delta t$. Then there exist $\gamma_{1}, \gamma_{2}, \gamma_{3}, \gamma_{4}$ and $\gamma_{5}$ such that the closedloop system has the following properties.

- $(x(t), y(t), z(t)) \in \mathcal{P}_{a}$ for all $t \geq 0$;

- $\lim _{t \rightarrow \infty}\left(x(t)-p_{r_{1}}(t)\right)=\lim _{t \rightarrow \infty}\left(y(t)-p_{r_{2}}(t)\right)=$ $\lim _{t \rightarrow \infty}\left(z(t)-p_{r_{3}}(t)\right)=0$.

Proof: To begin with, $q_{j}^{i}(t)<0$ for all $j \in\{1,2,3\}$ according to the definition of $m^{i}$. This proves the first property.

Consider the $i^{\text {th }}$ Lyapunov function (10) and note that $\dot{L}^{i}<0$ for all $m^{i} \neq 0, \theta^{i} \neq \theta^{i *}$ and $\phi^{i} \neq \phi^{i *}$. Consider now the switch from $u_{f}^{i}$ to $u_{f}^{j}$, with $i \neq j$, i.e. the group of active constraints changes. Without loss of generality, we consider switching from $L^{1}$ to $L^{2}$ at $t=t_{0}$. Note that

$$
\begin{array}{r}
L^{1}\left(t_{0}\right)=\frac{1}{2}\left[\begin{array}{r}
m_{1}^{2}\left(t_{0}\right)+m_{2}^{2}\left(t_{0}\right)+\left[\theta\left(t_{0}\right)-\theta^{1 *}\left(t_{0}\right)\right]^{2} \\
+m_{3}^{2}\left(t_{0}\right)+\left[\phi\left(t_{0}\right)-\phi^{1 *}\left(t_{0}\right)\right]^{2}
\end{array}\right], \\
L^{2}\left(t_{0}\right)=\frac{1}{2}\left[\begin{array}{r}
m_{1}^{2}\left(t_{0}\right)+m_{2}^{2}\left(t_{0}\right)+\left[\theta\left(t_{0}\right)-\theta^{2 *}\left(t_{0}\right)\right]^{2} \\
+m_{4}^{2}\left(t_{0}\right)+\left[\phi\left(t_{0}\right)-\phi^{2 *}\left(t_{0}\right)\right]^{2}
\end{array}\right],
\end{array}
$$

where $m_{1}=\log \frac{s_{1} p\left(t_{0}\right)+t_{1}}{s_{1} p_{r}\left(t_{0}\right)+t_{1}}, m_{2}=\log \frac{s_{2} p\left(t_{0}\right)+t_{2}}{s_{2} p_{r}\left(t_{0}\right)+t_{2}}$, $m_{3}=\log \frac{s_{3} p\left(t_{0}\right)+t_{3}}{s_{3} p_{r}\left(t_{0}\right)+t_{3}}$ and $m_{4}=\log \frac{s_{4} p\left(t_{0}\right)+t_{4}}{s_{4} p_{r}\left(t_{0}\right)+t_{4}}$ with $p=[x, y, z]^{T}$ and $p_{r}=\left[x_{r}, y_{r}, z_{r}\right]^{T}$. Since the time difference between two switches is not smaller than $\Delta t$, there always exists $\gamma_{1}, \gamma_{2}, \gamma_{3}, \gamma_{4}$ and $\gamma_{5}$ such that $\int_{t_{0}}^{t_{0}+\Delta t} \dot{L}^{2} d t \leq$ $L^{2}\left(t_{0}\right)-L^{1}\left(t_{0}\right)$, i.e. $L^{2}\left(t_{0}+\Delta t\right) \leq L^{1}\left(t_{0}\right)$. Suppose $\left\{i_{1}, i_{2}, \ldots, i_{I}\right\}$ is a sequence of active group of constraints, where $i_{j} \in\left\{1,2, \ldots, N_{c}\right\}$ for all $j \in\{1,2, \ldots, I\}$, and the $i_{j}^{\text {th }}$ group is active for the time period $\left(t_{i_{j}}, T_{i_{j}}\right]$ with $t_{i_{j+1}}=T_{i_{j}}$ for all $j \in\{1,2, \ldots, I-1\}$. Therefore $0 \leq$ $L^{i_{I}}\left(T_{i_{I}}\right)<\cdots<L^{i_{2}}\left(T_{i_{2}}\right)<L^{i_{1}}\left(T_{i_{1}}\right)$. Define the overall Lyapunov function $L(t)$ as

$$
L(t)=L^{i_{j}}(t), \text { if } t \in\left(t_{i_{j}}, T_{i_{j}}\right] .
$$

From the above analysis and [19] $L(t)$ is a multiple Lyapunov function and

$$
\begin{aligned}
& \lim _{t \rightarrow \infty}\left(x(t)-p_{r_{1}}(t)\right)=0, \quad \lim _{t \rightarrow \infty}\left(y(t)-p_{r_{2}}(t)\right)=0, \\
& \lim _{t \rightarrow \infty}\left(z(t)-p_{r_{3}}(t)\right)=0 .
\end{aligned}
$$

\section{B. Shared Control Theorem}

Similarly to [16] the overall set $\mathcal{R}$ can be divided into three subsets: the safe subset $\mathcal{R}_{s}$, the hysteresis subset $\mathcal{R}_{h}$ and the dangerous subset $\mathcal{R}_{d}$. Relative to the $i^{\text {th }}$ group of active constraints and a given $v_{h}$, the set $\mathcal{R}$ can be divided into $\tilde{\mathcal{R}}_{s}$, $\tilde{\mathcal{R}}_{h}$ and $\tilde{\mathcal{R}}_{d}$ by equations (12), given on the top of next page, where $e=[\cos \theta \cos \phi, \sin \theta \cos \phi, \sin \phi]^{T}, \mathcal{Q}_{a}^{i}=S^{i} \mathcal{P}_{a}+T^{i}$, and $b_{2}>b_{1}>0$. To eliminate the ambiguity for different groups of active constraints, we can pull back the sets $\tilde{\mathcal{R}}_{s}$, $\tilde{\mathcal{R}}_{h}$ and $\tilde{\mathcal{R}}_{d}$ into the $(x, y, z, \theta, \phi)$ coordinates using the equations

$$
\begin{aligned}
& \mathcal{R}_{s}^{i}\left(v_{h}\right)=\operatorname{diag}\left(\mathrm{S}^{\mathrm{i}}-1, \mathrm{I}\right)\left(\tilde{\mathcal{R}}_{\mathrm{s}}^{\mathrm{i}}-\operatorname{col}\left(\mathrm{T}^{\mathrm{i}}, 0\right)\right), \\
& \mathcal{R}_{h}^{i}\left(v_{h}\right)=\operatorname{diag}\left(\mathrm{S}^{\mathrm{i}-1}, \mathrm{I}\right)\left(\tilde{\mathcal{R}}_{\mathrm{h}}^{\mathrm{i}}-\operatorname{col}\left(\mathrm{T}^{\mathrm{i}}, 0\right)\right), \\
& \mathcal{R}_{d}^{i}\left(v_{h}\right)=\operatorname{diag}\left(\mathrm{S}^{\mathrm{i}}-1, \mathrm{I}\right)\left(\tilde{\mathcal{R}}_{\mathrm{d}}^{\mathrm{i}}-\operatorname{col}\left(\mathrm{T}^{\mathrm{i}}, 0\right)\right) .
\end{aligned}
$$

Note that, by construction, the subsets $\mathcal{R}_{s}, \mathcal{R}_{h}$ and $\mathcal{R}_{d}$ have the following properties:

- $\mathcal{R}_{s}^{i}\left(v_{h}\right) \cup \mathcal{R}_{h}^{i}\left(v_{h}\right) \cup \mathcal{R}_{d}^{i}\left(v_{h}\right)=\mathcal{R}\left(v_{h}\right)$ for all $i \in$ $\left\{1,2, \ldots, N_{c}\right\}$;

- $\mathcal{R}_{d}^{i}\left(v_{h}\right) \cap \mathcal{R}_{d}^{j}\left(v_{h}\right)=\emptyset$ for all $i \neq j$ and $i, j \in$ $\left\{1,2, \ldots, N_{c}\right\}$;

- $\mathcal{R}_{d}\left(v_{h}\right)=\mathcal{R}_{d}^{1}\left(v_{h}\right) \cup \cdots \cup \mathcal{R}_{d}^{N_{c}}\left(v_{h}\right)$,

$\mathcal{R}_{h}\left(v_{h}\right)=\mathcal{R}_{h}^{1}\left(v_{h}\right) \cup \cdots \cup \mathcal{R}_{h}^{N_{c}}\left(v_{h}\right)$,

$\mathcal{R}_{s}\left(v_{h}\right)=\mathcal{R}_{s}^{1}\left(v_{h}\right) \cap \cdots \cap \mathcal{R}_{s}^{N_{c}}\left(v_{h}\right)=\mathcal{R} \backslash\left(\mathcal{R}_{d} \cup \mathcal{R}_{h}\right)$.

The sharing-function relative to the $i^{\text {th }}$ group of active constraints $k^{i}$ can be defined as

$$
k^{i}\left(p, \theta, \phi, v_{h}\right)= \begin{cases}1, & (p, \theta, \phi) \in \mathcal{R}_{s}^{i}\left(v_{h}\right) \backslash \mathcal{R}_{d}^{i}\left(v_{h}\right), \\ l^{i}, & (p, \theta, \phi) \in \mathcal{R}_{h}^{i}\left(v_{h}\right) \\ 0, & (p, \theta, \phi) \in \mathcal{R}_{d}^{i}\left(v_{h}\right)\end{cases}
$$

where

$$
l^{i}= \begin{cases}1, & \text { if }(p, \theta, \phi) \text { enters } \mathcal{R}_{h}^{i}\left(v_{h}\right) \text { from } \mathcal{R}_{s}^{i}\left(v_{h}\right), \\ 0, & \text { if }(p, \theta, \phi) \text { enters } \mathcal{R}_{h}^{i}\left(v_{h}\right) \text { from } \mathcal{R}_{d}^{i}\left(v_{h}\right) .\end{cases}
$$

Finally, the shared-control input is given by

$$
u_{s}\left(p, \theta, \phi, v_{h}\right)=\sum_{i=1}^{N_{c}}\left[\left(1-k^{i}\right) u_{f}^{i}\right]+\left(\min _{i=1}^{N_{c}} k^{i}\right) u_{h} .
$$

Proposition 1: Consider the system (1) with the sharedcontroller (11)-(13)-(14). Let $\mathcal{P}_{a}$ be a closed and compact set described by (3) and $u_{h}$ be a given h-control. Assume $(x(0), y(0), z(0)) \in \mathcal{P}_{a}$ and the time that a feedback controller is active is larger than $\Delta t$. Then there exist $\gamma_{j}>0$, 


$$
\begin{gathered}
\tilde{\mathcal{R}}_{s}^{i}\left(v_{h}\right)=\left\{\left(q^{i}, \theta^{i}, \phi^{i}\right) \in \mathcal{Q}_{a}^{i} \times \mathbb{S} \times \mathbb{A}: s_{j}^{i} e v_{h} \leq \frac{1}{q_{j}^{i}+b_{2}}-\frac{1}{b_{2}} \text { if } q_{j}^{i} \geq-b_{2} \text { for all } j \in\{1,2,3\}\right\} \\
\tilde{\mathcal{R}}_{h}^{i}\left(v_{h}\right)=\left\{\begin{aligned}
&\left(q^{i}, \theta^{i}, \phi^{i}\right) \in \mathcal{Q}_{a}^{i} \times \mathbb{S} \times \mathbb{A}: \exists j \in\{1,2,3\} \text { such that } s_{j}^{i} e v_{h}>\frac{1}{q_{j}^{i}+b_{2}}-\frac{1}{b_{2}} \text { and } q_{j}^{i} \geq-b_{2} \\
& \text { and } s_{k}^{i} e v_{h}<\frac{1}{q_{k}^{i}+b_{1}}-\frac{1}{b_{1}} \text { if } q_{k}^{i} \geq-b_{1} \text { for all } k \in\{1,2,3\}
\end{aligned}\right\} \\
\tilde{\mathcal{R}}_{d}^{i}\left(v_{h}\right)=\left\{\begin{array}{cl}
\left(q^{i}, \theta^{i}, \phi^{i}\right) \in \mathcal{Q}_{a}^{i} \times \mathbb{S} \times \mathbb{A}: & \exists j \in\{1,2,3\} \text { such that } s_{j}^{i} e v_{h} \geq \frac{1}{q_{j}^{i}+b_{1}}-\frac{1}{b_{1}},-b_{1} \leq q_{j}^{i}<0 \\
& \text { or } \exists j \in\{1,2,3\} \text { such that } s_{j}^{i} e v_{h}>\frac{1}{q_{j}^{i}+b_{1}}-\frac{1}{b_{1}}, q_{j}^{i}=0 \\
& \text { or } \forall j \in\{1,2,3\} \text { such that } q_{j}^{i}=s_{j}^{i} e v_{h}=0
\end{array}\right\}
\end{gathered}
$$

for $j \in\{1,2,3,4,5\}$, and $b_{2}>b_{1}>0$ such that the sclosed-loop system has the following properties.

(1) $\Omega_{s}=\Pi_{\mathcal{R}_{s}}\left(\Omega_{h}\right)$.

(2) $(x(t), y(t), z(t)) \in \mathcal{P}_{a}$ for all $t \geq 0$.

(3) $u_{s}(t)=u_{h}(t)$ for all $t \geq 0$ and $(p(t), \theta(t), \phi(t)) \in$ $\mathcal{R}_{s}\left(v_{h}(t)\right)$.

Proof: Claim (1) is a consequence of the general results in [20], and of the fact that $\Pi_{\mathcal{R}_{s}}\left(\Omega_{h}\right)$ is the $\Omega$-limit set of both the h-closed-loop and the f-closed-loop systems (by definition, the former, and by equations (5)-(10), the latter).

As detailed in Section III-A, the f-control is such that the configuration of the system stays in the admissible region $\mathcal{P}_{a}$. In addition, as detailed in [16], any trajectory should enters the dangerous subset $\mathcal{R}_{d}$ before leaving $\mathcal{R}$ where $u_{f}$ is active. Therefore, the set $\mathcal{R}$ is forward invariant and claim (2) holds.

Finally, claim (3) is a direct consequence of the definition of the shared-control.

\section{CASE STUDY}

In this section we discuss a case study in which the configuration of the system needs to track a spiral. Note that the Cartesian position of the $\mathrm{h}$-closed-loop system goes outside of $\mathcal{P}_{a}$, i.e. $\Omega_{h} \not \subset \mathcal{R}_{s}$.

Consider the system (1) and the admissible configuration set defined by

$$
\mathcal{P}_{a}=\{(x, y, z) \mid x \geq 0, y \geq 0, z \geq 0\} .
$$

Assume the reference trajectory is a spiral described by

$$
p_{d}(t)=[3 \cos (0.1 t)+5,3 \sin (0.1 t)+4,0.1+0.02 t]^{T},
$$

and the h-control is a stable tracking controller.

Simulation results are given in Figure 2 to Figure 3. Figure $2 \mathrm{a}$ and Figure $2 \mathrm{~b}$ show that the configuration of the UAV for the h-closed-loop goes outside the admissible set (the grey shaded area), i.e. $x \leq 0$, while that for the s-closed-loop remains in the admissible set $\mathcal{P}_{a}$. Note that the projections

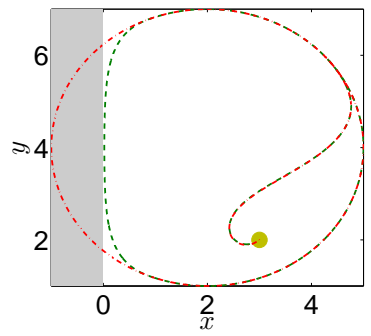

(a) $(x, y)$ paths

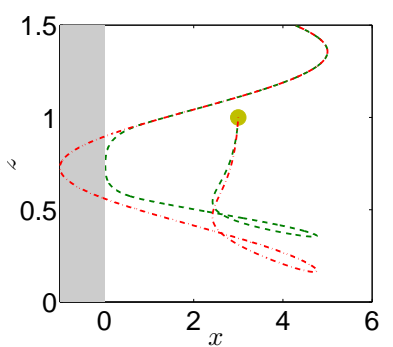

(b) $(x, z)$ paths

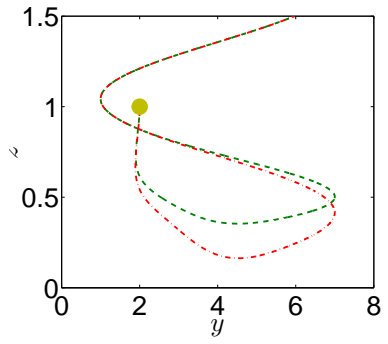

(c) $(y, z)$ paths

Fig. 2: Projections of paths of the system (1)in different planes for the set $\mathcal{P}_{a}$ given in (15) with $r=3, b_{2}=2, b_{1}=$ 1: the h-closed-loop (red, dash-dotted) and the s-closed-loop (green, dashed). Round mark: the initial position of the UAV.

of the trajectories of the h-closed-loop system and of the s-closed-loop system in the $x-z$ and $y-z$ plane do not overlap with each other in the beginning because the system states enters $\mathcal{R}_{d}$ where $u_{f}$ is active at the very beginning. Figure 3 displays the time histories of the bounded sharedcontrol inputs $u_{s}$ and of the state variables. It clearly shows that the input signals $v_{s}, \omega_{1 s}$ and $\omega_{2 s}$ are continuous and smooth functions and equal to $v_{h}, \omega_{1 h}$ and $\omega_{2 h}$, respectively, except for the time when the state of the system enters the dangerous subset $\mathcal{R}_{d}$.

\section{CONCLUSIONS}

We have proposed a solution to the shared-control problem for a UAV moving in the 3D space. The shared-control algorithm is based on a hysteresis switch and its formal 

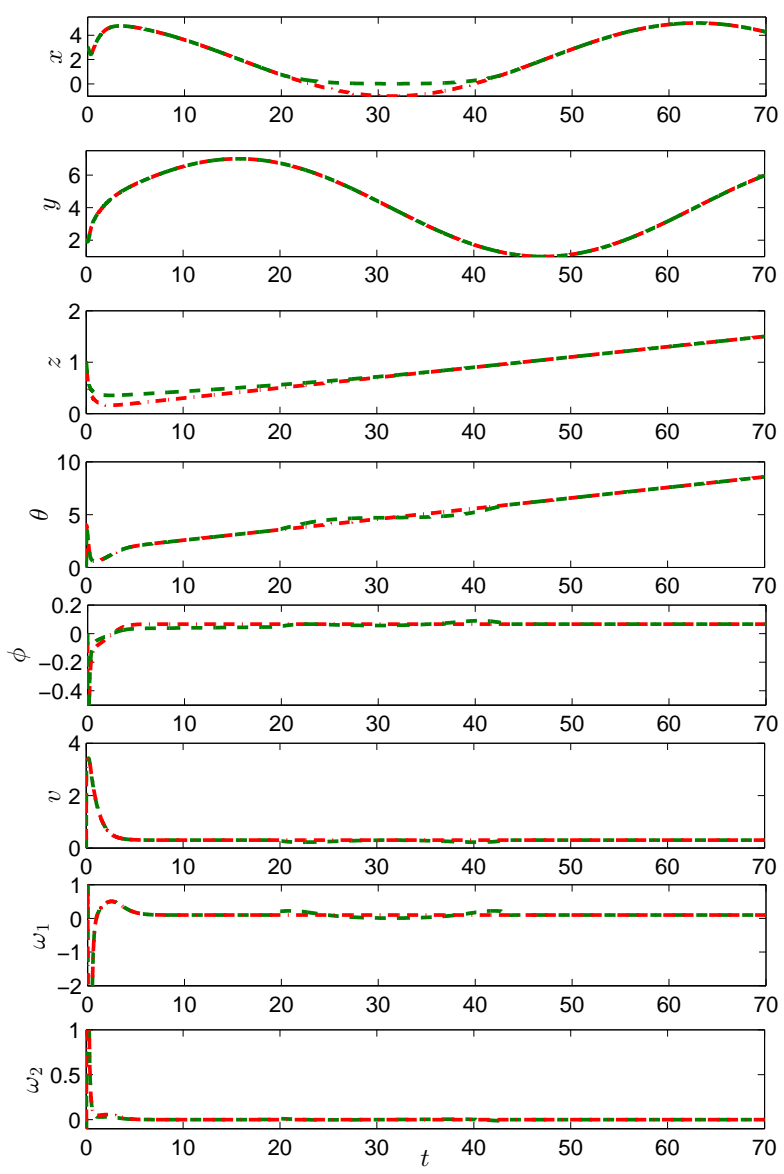

Fig. 3: Time histories of the variable $x, y, z, \theta, \phi, v, \omega_{1}$ and $\omega_{2}$ for the h-closed-loop system (red, dash-dotted) and the s-closed-loop system (green, dashed).

properties are established by a Lyapunov-like analysis. Two numerical examples are given in Section IV to show the effectiveness of the shared-control law. Future research will focus on the shared-control problem for multi-agent systems.

\section{REFERENCES}

[1] B. Yenne, Attack of the Drones: A History of Unmanned Aerial Combat. Zenith Press, 2004.

[2] M. Ma'sum, M. Arrofi, G. Jati, F. Arifin, M. Kurniawan, P. Mursanto, and W. Jatmiko, "Simulation of intelligent unmanned aerial vehicle (UAV) for military surveillance," in Proc. of International Conference on Advanced Computer Science and Information Systems, 2013, pp. 161-166.

[3] R. Beard, T. McLain, D. Nelson, D. Kingston, and D. Johanson, "Decentralized cooperative aerial surveillance using fixed-wing miniature UAVs," in Proc. of IEEE, 2006, pp. 1306-1324.

[4] J. Chen, "UAV-guided navigation for ground robot tele-operation in a military reconnaissance environment," Ergonomics, vol. 53, no. 8, pp. 940-950, 2010.

[5] K. Cook, "The silent force multiplier: The history and role of UAVs in warfare," in Proc. of IEEE Aerospace Conference, 2007, pp. 1-7.
[6] F. Mohammed, A. Idries, N. Mohamed, J. Al-Jaroodi, and I. Jawhar, "Opportunities and challenges of using UAVs for dubai smart city," in Proc. of International Conference on New Technologies, Mobility and Security, 2014, pp. 1-4.

[7] S. Zhou, Y. Wang, S. Ye, G. Zhu, and L. Cheng, "The intelligent wind detection system of weather UAV based on the multi-mode variable structure," in Proc. of International Conference on Intelligent Computation Technology and Automation, 2011, pp. 765-768.

[8] Z. Lin, "UAV for mappinglow altitude photogrammetric survey," International Archives of the Photogrammetry, Remote Sensing and Spatial Information Sciences, vol. XXXVII, no. Part B1, pp. 11831186, 2008

[9] G. Grenzdrffer, A. Engel, and B. Teichert, "The photogrammetric potential of low-cost UAVs in forestry and agriculture," International Archives of the Photogrammetry, Remote Sensing and Spatial Information Sciences, vol. XXXVII, pp. 1207-1214, 2008.

[10] T. Sangyam, P. Laohapiengsak, W. Chongcharoen, and I. Nilkhamhang, "Path tracking of UAV using self-tuning PID controller based on fuzzy logic," in Proc. of SICE Annual Conference, 2010, pp. $1265-1269$.

[11] Q. Zhou, Y. Zhang, C. Rabbath, and D. Theilliol, "Design of feedback linearization control and reconfigurable control allocation with application to a quadrotor UAV," in Proc. of Conference on Control and Fault-Tolerant Systems, 2010, pp. 371-376.

[12] M. Olivares-Mendez, I. Mondragon, P. Campoy, and C. Martinez, "Fuzzy controller for uav-landing task using 3d-position visual estimation," in Proc. of IEEE Internation Conference on Fuzzy Systems, 2010, pp. 1-8.

[13] L. Doitsidis, K. Valavanis, N. Tsourveloudis, and M. Kontitsis, "A framework for fuzzy logic based uav navigation and control," in Proc. of IEEE Internation Conference on Robotics and Automation, 2004, pp. 4041-4046.

[14] T. Dierks and S. Jagannathan, "Output feedback control of a quadrotor UAV using neural networks," IEEE Trans. on Neural Networks, vol. 21 , no. 1 , pp. 50-66, 2010.

[15] M. Huang, B. Xian, D. C., K. Yang, and Y. Feng, "Adaptive tracking control of underactuated quadrotor unmanned aerial vehicles via backstepping," in Proc. of American Control Conference, 2010, pp. 2076-2081.

[16] J. Jiang and A. Astolfi, "Shared-control for the kinematic model of a mobile robot," in Proc. of IEEE Conference on Decision and Control, Los Angeles, USA, 2014, pp. 62-67.

[17] C. Byrnes, F. Celani, and A. Isidori, "Omega-limit sets of a class of nonlinear systems that are semiglobally practically stabilized," International Journal of Robust and Nonlinear Control, vol. 15, no. 7, pp. 315-333, 2005.

[18] J. Jiang and A. Astolfi, "Shared-control for fully actuated linear mechanical systems," in Proc. of IEEE Conference on Decision and Control, Florence, Italy, 2013, pp. 4699-4704.

[19] D. Liberzon, Switching in Systems and Control. Birkhauser, Boston, 2003.

[20] C. Prieur, "Uniting local and global controllers with robustness to vanishing noise," Math. Control Signals Systems, vol. 14, pp. 143172, 2001. 